\title{
CARBON AND SILICON IN NORMAL A STARS AND IN LAMBDA BOOTIS STARS
}

\section{HARTMUT HOLWEGER and SVEN STÜRENBURG}

Institut für Theoretische Physik und Sternwarte, Universität Kiel, Olshausenstrasse 40, W-2300 Kiel, Federal Republic of Germany

\begin{abstract}
The anticorrelation of $\mathrm{C}$ and $\mathrm{Si}$ previously inferred from high-resolution spectroscopy of sharp-lined A stars classified as normal or mild Am is also found among the metal-deficient, more rapidly rotating $\lambda$ Boo stars. The entire sample of program stars covers the range $[\mathrm{C} / \mathrm{Si}]=-1.14$ ( Sirius, mild Am star ) to +1.35 ( HR 7400, $\lambda$ Boo-type ). Iron varies parallel with silicon. With respect to surface chemistry the $\lambda$ Boo stars do not form a distinct group but rather represent the lower end in a continuous sequence of 'metal' abundance extending over more than 1.5 dex.

We argue that differential accretion of gas and dust in late stages of star formation or during the main-sequence phase is responsible for most of the abundance variations of the lighter elements up to the iron group. Enhanced or impeded accretion of dust leads to a. metal-rich or metal-poor surface composition, respectively. The heavy trace elements $\mathrm{Sr}$ and $\mathrm{Ba}$ show additional anomalies indicating that other processes like gravitational and radiative separation are also involved.
\end{abstract}

\section{INTRODUCTION}

The present study is part of a program intended to establish a homogenous set of abundance data for main-sequence stars of early spectral type $A$. The observational basis consists of high resolution Reticon and CCD spectra. Model-atmosphere analysis is carried out in the same manner for all stars of the program.

It was hoped that this differential procedure would permit to identify compositional differences or regularities possibly occurring among nonmagnetic, non-exotic A stars. In a foregoing paper (Holweger 1992; paper I) attention was drawn to a well-defined anticorrelation of carbon and silicon found to exist among A stars classified as normal. The present study focuses on their metal-poor counterparts, the $\lambda$ Boo stars. 


\section{PROGRAM STARS AND OBSERVATIONS}

The sample of normal $A$ stars comprises all sharp-lined ( $\mathrm{v} \sin \mathrm{i}<50$ $\mathrm{km} / \mathrm{s}$ ) B9.5 - A2 V stars classified as normal (i.e. with no known pecularities) in the Bright Star Catalogue (Hoffleit \& Jaschek 1982) which are single stars or widely separated binaries and are observable from La Silla, Chile.

Our selection of Lambda Bootis stars is based on the revised classification by Gray (1988). Spectra have been obtained for 14 out of the 16 objects catalogued by Gray.

The observations consist of Reticon and CCD spectra recorded with the ESO Coude Echelle Spectrometer at the $1.4 \mathrm{~m}$ CAT. A resolving power of 50000 was used throughout. Three of the $\lambda$ Boo stars were observed at DSAZ, Calar Alto (Spain) using the long-focus Coudé Camera + CCD at the $2.2 \mathrm{~m}$ telescope. For Vega, broad-band, highdispersion photographic spectra recorded at Mount Wilson Observatory were kindly made available to us prior to publication (Griffin \& Griffin 1992). All these spectra have comparable spectral resolution.

\section{ANALYSIS}

\section{Normal A stars}

An overview of the observations and a first assessment of obvious chemical anomalies has been given by Holweger et al. (1986). Detailed model atmosphere analyses taking into account non-LTE effects for certain elements have been published (Gigas 1986, 1988; Lemke 1989, 1990). The non-solar composition of Vega and the other 'normal' A stars has become obvious. Results of the quoted papers pertinent to the present study are listed in Table 1.

\section{Lambda Bootis stars}

A selection of spectra is depicted in Holweger and Stürenburg (1991); one of the objects has turned out to be a possible $\beta$ Pic-type shell star. Preliminary results of a model-atmosphere analysis have been reported (Stürenburg 1992a); a comprehensive report will be published elsewhere (Stürenburg 1992b). Here we present results for $\mathrm{C}, \mathrm{Si}$, and $\mathrm{Fe}$ which we discuss with respect to the anticorrelation of $\mathrm{C}$ and $\mathrm{Si}$ found among the normal A stars (paper I).

The method of analysis is the same as employed for the normal A stars. However, strong rotational broadening makes single-line analysis difficult or impossible and requires detailed spectrum synthesis. Some elements are represented by lines which are sensitive to microturbulence. Microturbulent velocities were determined by comparing abundances derived from lines of different strength; species suitable for this purpose are mainly $\mathrm{Fe}$ I and in some cases also Fe II and Ti II. Effective temperatures and surface gravities have been derived from Strömgren 
TABLE I The program stars: basic data and derived abundances

\begin{tabular}{cccccccccc}
\hline \multicolumn{7}{c}{ Normal A stars } \\
\hline HR & name & $T_{\text {eff }}$ & $\log g$ & $v \cdot \sin i$ & {$[\mathrm{C} / \mathrm{H}]$} & {$[\mathrm{Si} / \mathrm{H}]$} & {$[\mathrm{C} / \mathrm{Si}]$} & {$[\mathrm{Fe} / \mathrm{H}]$} \\
\hline 1448 & & 9200 & 3.70 & 24 & -0.11 & -0.06 & -0.05 & +0.24 \\
1975 & & 10170 & 4.50 & 33 & -0.23 & +0.28 & -0.51 & +0.11 \\
2491 & Sirius & 9900 & 4.30 & 16 & -0.76 & +0.38 & -1.14 & +0.33 \\
3136 & & 9650 & 4.00 & 35 & -0.77 & +0.17 & -0.94 & +0.35 \\
4138 & & 9300 & 3.65 & 6.5 & -0.02 & -0.42 & +0.40 & +0.23 \\
4359 & $\theta$ Leo & 9460 & 3.45 & 21 & -0.16 & +0.15 & -0.31 & +0.43 \\
5798 & & 9900 & 3.70 & 38 & +0.31 & -0.57 & +0.88 & -0.08 \\
6070 & & 9600 & 4.50 & 36 & -0.21 & +0.21 & -0.42 & +0.07 \\
7001 & Vega & 9500 & 3.90 & 23 & -0.30 & -0.61 & +0.31 & -0.43 \\
7773 & $\nu$ Cap & 10300 & 3.80 & 22 & -0.26 & +0.21 & -0.47 & +0.08 \\
8576 & $\beta$ PsA & 9600 & 4.20 & 26 & -0.03 & +0.25 & -0.28 & +0.07 \\
\hline & & & Lambda Bootis stars & & & \\
\hline HR & name & $T_{\text {eff }}$ & log $g$ & $v \cdot \sin i$ & {$[\mathrm{C} / \mathrm{H}]$} & {$[\mathrm{Si} / \mathrm{H}]$} & {$[\mathrm{C} / \mathrm{Si}]$} & {$[\mathrm{Fe} / \mathrm{H}]$} \\
\hline 12 & & 8140 & 3.80 & 60 & +0.05 & -0.45 & +0.50 & -0.57 \\
541 & & 7950 & 3.83 & 125 & +0.05 & -0.20 & +0.25 & -1.35 \\
1570 & $\pi^{1}$ Ori & 8970 & 4.25 & 120 & +0.05 & -0.60 & +0.65 & -0.75 \\
1989 & 131 Tau & 8970 & 3.60 & 200 & -0.15 & -0.30 & +0.15 & -0.02 \\
4881 & & 7450 & 3.93 & 135 & -0.30 & -1.00 & +0.70 & -1.35 \\
7400 & 35 Aql & 9260 & 4.22 & 90 & +0.25 & -1.10 & +1.35 & -1.15 \\
7736 & 29 Cyg & 7990 & 3.99 & 80 & -0.20 & -1.00 & +0.80 & -1.05 \\
$7764 \mathrm{C}$ & & 7860 & 3.74 & 240 & -0.10 & +0.00 & -0.10 & -0.60 \\
7959 & & 7970 & 3.98 & 200 & -0.25 & -0.20 & -0.05 & -0.70 \\
7960 & & 7970 & 3.98 & 180 & -0.25 & -0.20 & -0.05 & -0.70 \\
8203 & & 8100 & 4.03 & 65 & -0.40 & -0.55 & +0.15 & -0.82 \\
8437 & & 7450 & 3.75 & 55 & +0.00 & -0.65 & +0.65 & -0.97 \\
\hline \hline
\end{tabular}


photometry (catalogue of Hauck \& Mermilliod 1985) using the calibration of Moon \& Dworetsky (1985).

The basic parameters are listed in Table I together with the abundances of $\mathrm{C}, \mathrm{Si}$ and $\mathrm{Fe}$ (in logarithmic units relative to the Sun). The quoted abundances include non-LTE corrections for lines of C I and Fe I derived from the Kiel non-LTE code, employing model atoms described in Stürenburg \& Holweger (1990) and Steenbock \& Gehren (1992), respectively. For the stars and elements studied here the non-LTE abundance corrections are not critical and, moreover, of similar magnitude. Typical values are -0.07 dex for C I and +0.14 dex for Fe I. The latter include a 50\% reduction of the calculated values since Gigas (1986) and Lemke (1989) have found evidence that the Fe I model atom leads to overestimate the non-LTE corrections in A stars. Analogous corrections were applied to the normal A stars. For the solar iron abundance we have taken into account its recent downward revision, adopting $\log \mathrm{N}(\mathrm{Fe})=7.51$.

\section{Turbulence in A stars}

Spectroscopic determinations of microturbulence in the normal A stars yield average velocities of $2.2 \mathrm{~km} / \mathrm{s}$, with individual values ranging from 1.2 to $3.0 \mathrm{~km} / \mathrm{s}$ (Lemke 1989). Similar values were found in the $\lambda$ Boo stars. For all but the weakest lines microturbulence can have a profound effect on line formation and abundance determination (see the discussion of discrepant literature data by Lane and Lester 1987).

In spite of the clear spectroscopic signature of microturbulence the underlying dynamical processes have often be considered as being somewhat mysterious. In the case of the Sun numerical models of photospheric convection have helped to clearify the situation. In solartype stars microturbulence is just the small-scale part of the basic convective flow pattern (see e.g. Steffen and Freytag 1991). Preliminary results of a project to extend such numerical simulations towards the upper main sequence (Freytag 1992) suggest that this picture essentially remains valid up to effective temperatures of at least $8500 \mathrm{~K}$. A snapshot of a time series for an A5 star is presented in Fig. 1. In these computations a 2D cartesian geometry is assumed; radiative transfer is calculated in 3D, the diffusion approximation is avoided. Details of the code are decribed elsewhere (Steffen 1991, Steffen and Freytag 1991). During further development of the flow pattern its topology did not change much, but the velocities in the surface convection zone increased significantly over the value of $1.6 \mathrm{~km} / \mathrm{s}$ in the snapshot, reaching $9 \mathrm{~km} / \mathrm{s}$ in some places. Detailed line formation calculations are not yet available, but it is obvious that this vigorous velocity field will result in significant non-thermal line broadening.

Another interesting feature, pertinent to diffusion theory, is that a separation into a superficial $\mathrm{H}-\mathrm{He}$ convection layer and $\mathrm{He}^{+}$convection zone well below the surface never occurred in these models. 


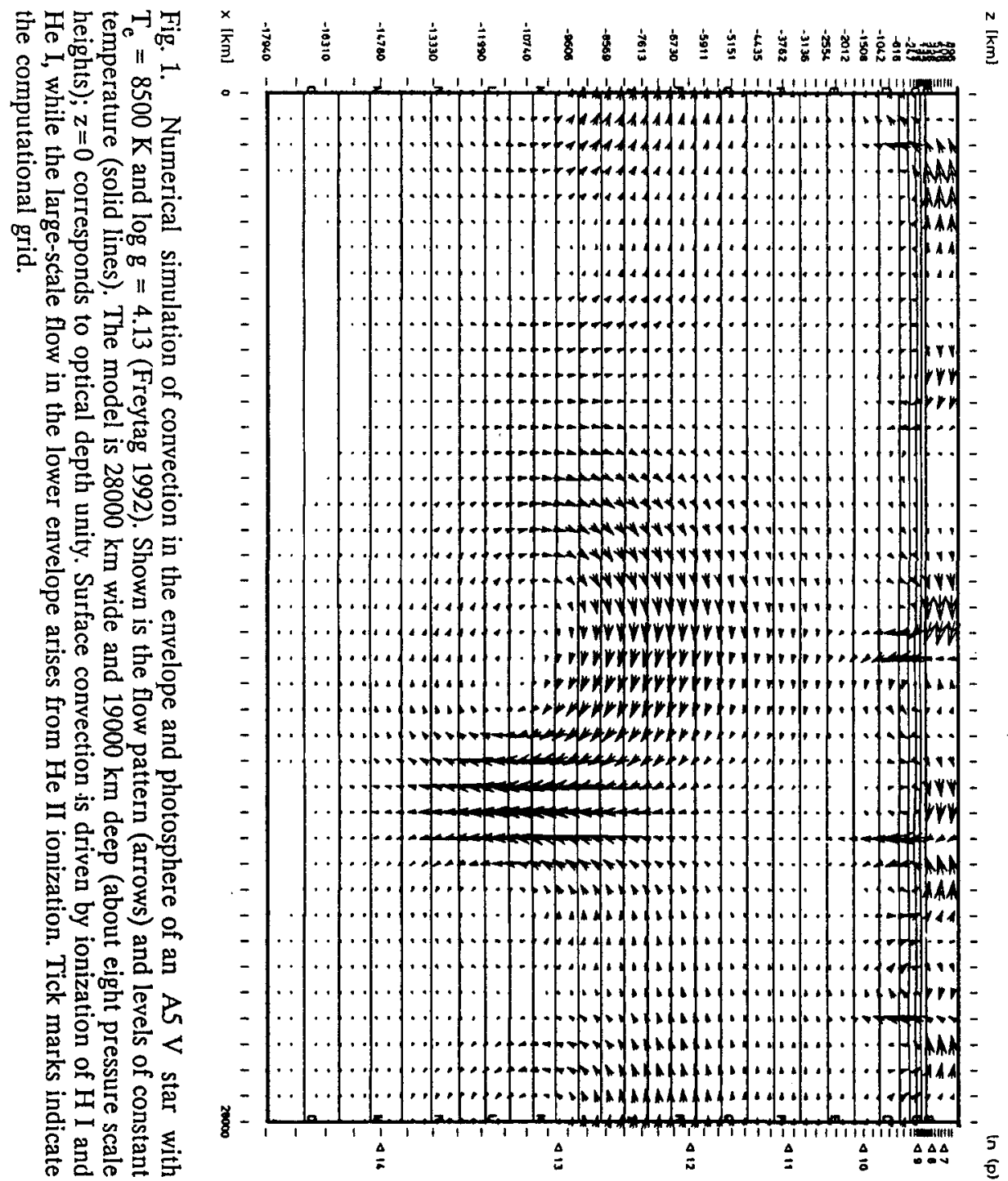




\section{RESULTS}

Fig. 2 shows the $[\mathrm{C} / \mathrm{Si}]$ and $[\mathrm{Si} / \mathrm{H}]$ data of the normal $\mathrm{A}$ stars already discussed in paper I supplemented by the new results for the $\lambda$ Boo stars; the filled diamonds designate Vega (upper left quadrant) and Sirius (bottom), respectively. The new data significantly extend the range of anomalies shown in Fig. 1 of paper I towards large [C/Si] values; at the same time it is obvious that there is a continuous sequence of [C/Si] extending from at least -0.5 to more than +1.0 . Fig. 1 of paper I also includes a typical hot Am star, o Peg, analyzed by Adelman (1988a,b) and two blue stragglers in the old open cluster M67 studied by Mathys (1991). If the corresponding data points are included the lower part of the [C/Si] sequence becomes more evenly populated and extends down to - 1.1, leaving Sirius still as the most extreme object on this side.

Taken together this strongly suggests that mild Am and $\lambda$ Boo stars are opposite extremes of a continuous sequence of anomalies interconnected by A stars classified as normal and by the less extreme members of the $\lambda$ Boo group. As already noticed by Lemke $(1989,1990)$ the variations from star to star of $[\mathrm{Si} / \mathrm{H}]$ are accompanied by parallel variations of iron-group elements like Ti and Fe. Fig. 3 demonstrates that this is true also for the $\lambda$ Boo stars, emphasizing the relationship between both classes of objects, although the correlation of $\mathrm{Fe}$ and $\mathrm{Si}$ appears to be less tight than the anticorrelation of $\mathrm{C}$ and $\mathrm{Si}$.

\section{DISCUSSION}

\section{[C/Si] as an indicator of gas-dust separation}

The peculiar behaviour of carbon in the normal A stars was tentatively attributed in paper I to its extremely low condensation temperature of less than $100 \mathrm{~K}$ (see e.g. Jenkins 1987 and references therein) as compared to more than $1200 \mathrm{~K}$ in the case of $\mathrm{Si}$ and $\mathrm{Fe}$. Carbon can remain in the gas phase in molecular form, mainly as $\mathrm{CO}$. This volatility is reflected in the comparatively small depletion of carbon (and of $\mathrm{N}, \mathrm{O}$, and $\mathrm{S}$ as well) in the interstellar gas. $\mathrm{Si}$ and $\mathrm{Fe}$, on the other hand, are almost completely condensed in solid form. The volatility of $\mathrm{C}$ is likely to be important not only in the interstellar medium, but also in the protostellar cloud or during later accretion phases. Its signature can be seen in the solar system: the most primitive meteorites, the CI chondrites, represent an early condensate which contains most elements in solar proportions, but C, N, and - to lesser extent - $\mathrm{O}$ are deficient. The $\mathrm{C} / \mathrm{Si}$ ratio in $\mathrm{Cl}$ chondrites is lower than the photospheric value by more than a factor of 10 (Holweger 1977). Obviously $\mathrm{C}$ and $\mathrm{N}$ have remained in the gas phase in the protosolar cloud during condensation of meteoritic matter. 

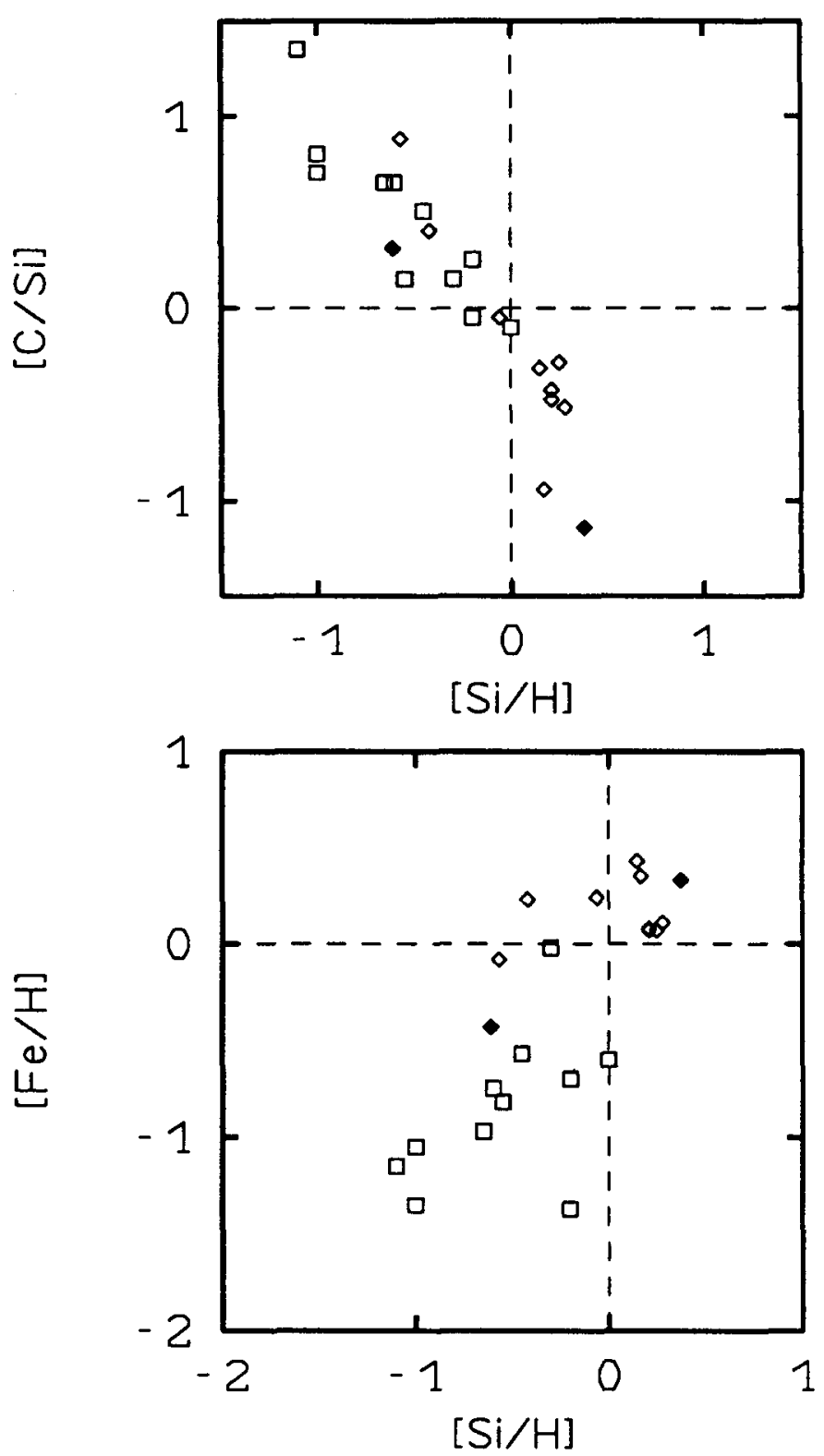

Fig. 2 (top). Anticorrelation of $[\mathrm{C} / \mathrm{Si}]$ and $[\mathrm{Si} / \mathrm{H}]$ in normal A stars (diamonds) and Lambda Bootis stars (squares). Dashed lines indicate solar abundance ratios.

Fig. 3 (bottom). Correlation of $[\mathrm{Fe} / \mathrm{H}]$ and $[\mathrm{Si} / \mathrm{H}]$. Symbols are the same as in Fig. 2. 
Any separation of gas and dust during star formation or later accretion phases will affect the abundance ratio of condensable elements like $\mathrm{Si}$ and $\mathrm{Fe}$, to volatiles like $\mathrm{H}, \mathrm{C}, \mathrm{N}$ and $\mathrm{O}$, leaving the $\mathrm{Fe} / \mathrm{Si}$ ratio essentially unaffected. In particular, preferential accretion of dust will lead to a metal-rich composition with $[\mathrm{Si} / \mathrm{H}]>0$ and $[\mathrm{C} / \mathrm{Si}]<0$ like that of $\theta$ Leo and Sirius, while preferential accretion of gas results in metaldeficient matter with a $\mathrm{C} / \mathrm{Si}$ ratio exceeding the solar value. Vega and the $\lambda$ Boo stars are candidates.

The idea that the balance of gas and dust during star formation may be a decisive factor regulating stellar 'metal' content has been conceived long ago (Whipple 1946; Schwarzschild et al. 1951) and subsequently taken up again in various investigations. In the comparison of solar and meteoritic matter quoted above it was concluded that in the case of the Sun no drastic pre-stellar fractionation of gas and dust has occured, but minor imbalance could be responsible for the spread in metallicity observed among cluster stars. Venn \& Lambert (1990) have proposed accretion of metal-deficient gas as an explanation for the abundance patterns of three $\lambda$ Boo stars and of Vega, a model which was worked out further by Charbonneau (1991) in the context of diffusion theory. Deviations from the normal gas-dust ratio of either sign were invoked in paper I to explain the anticorrelation of $\mathrm{C}$ and $\mathrm{Si}$ (and other elements) found among the normal $\mathrm{A}$ stars. We conclude that the continuous sequence of $[\mathrm{C} / \mathrm{Si}]$ values documented in Fig. 2 provides a strong argument in favour of a common explanation of the observed star-to-star variations in terms of a different degree, and direction, of gas-dustseparation.

How can separation of gas and dust occur, and why does it vary from star to star?

The physical mechanisms involved remain to be specified in the context of star formation and main-sequence evolution. In the case of the metalpoor (i.e. dust-deficient) $\lambda$ Boo stars Venn and Lambert (1990) have surveyed earlier work on interstellar clouds, but without a conclusive result. It appears that an elaborate study of the evolution of protostellar envelopes by Yorke (1979) has escaped notice, where the time-dependent separation of dust and gas through radiation pressure and its interplay with other processes is calculated explicitly for a model with $3 \mathrm{M}_{\odot}$ and $10 \mathrm{M}_{\odot}$, respectively. The coupling between charged grains and ionized gas is taken into account, it is of minor importance for the lower mass star. To quote Yorke ( p. 315, l.c.), 'the separation of dust and gas during "advanced" evolutionary stages (i.e. some time after the formation of the main sequence star) is a common phenomenon'. For the more massive model the resulting metal deficiency as a function of stellar radius is depicted in his Fig. 4.

These 1D calculations refer to the idealized case of spherical symmetry and necessarily include other simplifications as well. Rotation and 
turbulence are likely to reduce the predicted chemical anomalies in the stellar envelope to an extent that may vary from star to star, eventually leading to a distribution of metal deficiencies ranging from the extreme values observed in $\lambda$ Boo stars to the mild anomalies in some of the normal A stars.

However, the metal-rich A stars of our sample seem to require an additional mechanism which can lead to preferential accretion of dust rather than of gas by counteracting and overcoming radiative pressure. The most obvious such force is gravitation. Indeed, the discovery of a dust shell around Vega (Aumann et al. 1984) and other A stars implies that under certain circumstances dust can remain close to the star over much of its main sequence lifetime. As Aumann et al. argue particles larger than about $0.06 \mathrm{~cm}$ in radius will remain in orbit for $10^{8}$ years, while all particles smaller than $9 \mu \mathrm{m}$ - including interstellar grains - would have been blown away by radiation pressure. Aumann et al. conclude that very significant growth in particle size must have occurred since the time of formation of Vega. In view of the efficient radiative separation of interstellar grains and gas in the models of Yorke (1979) it seems likely that most of the particle growth has in fact occurred during late stages of star formation.

Particles of intermediate size, on the other hand, will spiral towards the star as a consequence of the Poynting-Robertson effect. If the growth of the particles in the dust shell of Vega had proceeded less far than postulated by Aumann et al. (1984) then we would expect them to have been incorporated into the stellar envelope during the final accretion phase, and Vega probably would not be so metal-deficient. Thus, depending on growth rate and final size distribution, the star may accrete a dust-rich or dust-deficient mixture of protostellar matter, leading to a corresponding over- or underabundance of 'metals' in its envelope.

Rotation may be an important parameter in this context. We note that the $\lambda$ Boo stars are rather rapid rotators, while the Am stars rotate slowly. The normal program stars have been selected to be sharp-lined, and it would be interesting to see if rapidly rotating $A$ stars with high metallicity, i.e. low [C/Si] exist at all.

\section{Lambda Bootis stars - normal A stars - Am stars}

Are the Am stars simply the metal-rich (i.e. dust-rich) counterpart of the $\lambda$ Boo group? Judged from $\mathrm{C}, \mathrm{Si}$, and $\mathrm{Fe}$ alone this possibility cannot easily be excluded, although the slope of the sequence in Fig. 2 appears to change towards the carbon deficient side (this becomes more obvious if three other recent determinations of $\mathrm{C}$ and $\mathrm{Si}$ are included - see paper I). However, the well-established overabundance with respect to iron of the heavy trace elements implies that other processes like gravitational and radiative separation may become important or dominant for certain elements. Their signature is clearly present among normal A stars as well, as demonstrated by Lemke (1990). In particular, his Fig. 1 indicates a 
systematic increase of $[\mathrm{Sr} / \mathrm{Fe}]$ and $[\mathrm{Ba} / \mathrm{Fe}]$ towards the metal-rich side, $[\mathrm{Fe} / \mathrm{H}]>0$. One possible interpretation is that in the metal-rich domain selective radiative and gravitational forces are important for $\mathrm{Sr}$ and $\mathrm{Ba}$, and also contribute to the abundance variation of $\mathrm{Fe}$ and other lighter elements in addition to gas-dust separation.

The most conspicuous examples are Sirius and HR 3136. In addition to the excess of iron they show the largest barium overabundance of all stars in our sample, $[\mathrm{Ba} / \mathrm{H}]=+1.4$ according to Lemke (1990). With respect to the lighter elements Sirius and HR 3136 are also peculiar by exhibiting a marked deficiency of carbon, $[\mathrm{C} / \mathrm{H}]=-0.8$, in contrast to the other stars.

\section{ACKNOWLEDGEMENTS}

We wish to thank Roger and Elizabeth Griffin for providing highdispersion spectrograms of Vega prior to publication. We are grateful to Bernd Freytag for his permission to quote unpublished results of numerical models of convection. This work was supported by the Deutsche Forschungsgemeinschaft under grant Ho 596/30-1.

\section{REFERENCES}

Adelman S., 1988a, MNRAS 230, 671

Adelman S., 1988b, MNRAS 235, 749

Aumann H.H., Gillett F.C., Beichman C.A., de Jong T., Houck J.R., Low F.J., Neugebauer G., Walker R.G., Wesselius P.R., 1984, ApJ 278, L23

Charbonneau P., 1991, ApJ 372, L33

Freytag B., 1992, in preparation

Gigas D., 1986, A\&A 165, 170

Gigas D., 1988, A\&A 192, 264

Gray R.O., 1988, AJ 95, 220

Griffin R.E.M., Griffin R.F., 1992, A photometric atlas of the spectrum of Vega, in preparation

Hauck B., Mermilliod M., 1985, A\&AS 60, 61

Hoffleit D., Jaschek C., 1982, The bright star catalogue,

Yale University Observatory, New Haven

Holweger H., 1977, Earth Planet Sci. Letters 34, 152

Holweger H., 1992, in: The Atmospheres of Early-Type Stars, eds. U. Heber, C.S. Jeffery, Springer, Heidelberg, p. 48 (paper I)

Holweger H., Steffen M., Gigas D., 1986, A\&A 163, 333

Holweger H., Stürenburg S., 1991, A\&A 252, 255

Jenkins E.B., 1987, in: Interstellar Processes, eds. D.J. Hollenbach,

H.A. Thronson, Jr., Reidel, Dordrecht, p. 533 
Lane M.C., Lester J.B., 1987, ApJS 65, 137

Lemke M., 1989, A\&A 225, 125

Lemke M., 1990, A\&A 240, 331

Mathys G., 1991, A\&A 245, 467

Moon T.T., Dworetsky M.M., 1985, MNRAS 217, 305

Schwarzschild M., Spitzer, Jr. L, Wildt R., 1951, ApJ 114, 398

Steffen M., Freytag B., 1991, in: Reviews in Modern Astronomy 4, Springer, Heidelberg, p. 43

Steffen M., 1991, in: Stellar Atmospheres: Beyond Classical Models, eds. L. Crivellari, I. Hubeny, D.G. Hummer, NATO ASI series, Kluwer, Dordrecht, p. 247

Steenbock W., Gehren T., 1992, A\&A, submitted

Stürenburg S., 1992a, in: The Atmospheres of Early-Type Stars, eds. U. Heber, C.S. Jeffery, Springer, Heidelberg, p. 228

Stürenburg S., 1992b, in preparation

Stürenburg S., Holweger H., 1990, A\&A 237, 125

Venn K.A., Lambert D.L., 1990, ApJ 363, 234

Whipple F.L., 1946, ApJ 104, 1

Yorke H.W., 1979, A\&A 80, 308 